\title{
A Symbolic Manipulation Approach for Modelling and Performance Analysis of Flexible Manipulator Systems
}

\author{
Z. Mohamed and M.O. Tokhi ${ }^{\dagger}$ \\ Department of Automatic Control and Systems Engineering, The University of Sheffield, UK
}

(Received 7 November 2000; accepted 17 August 2001)

\begin{abstract}
This paper presents a symbolic manipulation approach for modelling and performance analysis of a flexible manipulator system using finite element methods. A constrained planar single-link flexible manipulator is considered. A symbolic algorithm characterising the dynamic behaviour of the system is developed using a symbolic language. Using this approach, the system transfer functions are obtained in symbolic forms. Analyses and investigations in terms of system stability, time response to an input command and vibration frequencies are presented. Numeric and experimental results are presented for validation and assessment of the symbolic model.
\end{abstract}

${ }^{\dagger}$ Member of the International Institute of Acoustics and Vibration (IIAV)

\section{INTRODUCTION}

Flexible robot manipulators exhibit several advantages over their rigid counterparts: they require less material, are lighter in weight, consume less power, require smaller actuators, are more manoeuvrable and transportable, have less overall cost and higher payload to robot weight ratio. ${ }^{1}$ However, control of flexible manipulators to maintain accurate positioning is an extremely challenging problem. Due to the flexible nature of the system, the dynamics are highly nonlinear and complex. Problems arise due to difficulties in sensing the end-point displacement, vibration due to system flexibility, precise positioning requirement and the difficulty in obtaining an accurate model for the system. ${ }^{2,3}$ Therefore, flexible manipulators have not been favoured in production industries, due to un-attained end-point positional accuracy in response to input commands. Thus, a control mechanism that accounts for both rigid body and flexural motions of the system is required.

The complexity of this problem increases dramatically when a flexible manipulator carries a payload. In practice, robots are required to perform a single or sequential task such as picking up a payload, move it to a specified location or along a pre-planned trajectory and placing the payload. Previous investigations have shown that the dynamic behaviour of the manipulator is affected significantly by payload variations. ${ }^{4,5}$ Thus, the effects of payload on the dynamic characteristics of the manipulator have to be studied and identified. If the advantages associated with lightness are not to be sacrificed, accurate models and efficient controllers have to be developed.

Various approaches have been developed previously for the modelling of flexible manipulators. ${ }^{1}$ These include assumed modes, ${ }^{6-8}$ finite difference (FD) ${ }^{9}$ and finite element $(\mathrm{FE})^{\mathbf{1 0 - 1 2}}$ methods to solve the partial differential equation characterising the dynamic behaviour of a flexible manipulator system. Previous investigations have shown that the FE method can be utilised to obtain a good representation of the system. It has also been reported that in using FE methods, a single element is sufficient to describe the dynamic behaviour of a flexible manipulator reasonably well. Using a single element, the first two modes of vibration are described well. ${ }^{12}$ Moreover, the FE method exhibits several advantages over the FD method. ${ }^{10}$

Most of the investigations involving the FE method are numerical-based. Dynamic characteristics of the manipulator including stability, time response and vibration frequencies are interpreted on the basis of a single particular case, with no provision for any generality. Moreover, numerical systems must operate using numeric approximations, whose precision is limited by the computer hardware. Alternatively, exact quantities can be obtained by retaining the computations in a symbolic form. A distinguishing feature of symbolicbased methods is the mathematically comprehensive output they generate, allowing the significance of individual terms, or group of terms, to be identified. This brings with it the opportunity to gain insights into the model that would otherwise not be available. A symbolic manipulation will open up the possibility of analysing a system in both new and interesting ways. It can be seen that the trend over time has been away from fully numeric methods of formulation towards those with a strong and total symbolic flavour to them. This is due to the overwhelmingly rapid improvements in computer hardware technology in general and in computer algebra software in particular. ${ }^{\mathbf{1 3}}$

Symbolic approaches for modelling and simulation of flexible manipulators have previously been investigated. Most of these investigations have developed automated symbolic derivations of dynamic equations of motion of rigid and flexible manipulators utilising Lagrangian formulation and assumed mode methods, ${ }^{14-16}$ Hamilton's principle and nonlinear integro-differential equations ${ }^{17}$ and FD approximations. ${ }^{18}$ These have demonstrated that the approach has some advantages, such as allowing independent variation of flexure parameters. However, in utilising this manipulation approach, not much work has been done on the modelling and analysis of a flexible manipulator using FE methods. Moreover, relations between system parameters including payload and hub inertia and the system characteristics have not been ade- 